\title{
Neuroinfección por Listeria monocytogenes en una paciente pediátrica con leucemia aguda linfoblástica de reciente diagnóstico. La relevancia del diagnóstico precoz
}

\author{
Listeria monocytogenes neuroinfection in a pediatric patient with newly \\ diagnosed acute lymphoblastic leukemia. The relevance of early diagnosis
}

\author{
Abel Valderrama Cisneros,* Nathaly Navarro Parada, ${ }^{\ddagger}$ John Lopera Marín, ${ }^{\S}$ Laura Castañeda Correa ${ }^{\llbracket}$ \\ * Pediatra, Neurólogo Pediatra. Universidad Nacional Autónoma de México, Docente de la Fundación \\ Universitaria de Ciencias de la Salud, Coordinador del Servicio de Neurología Pediátrica. \\ ‡ Pediatra. Hospital Infantil Universitario de San José, Fundación Universitaria de Ciencias de la Salud, Bogotá, Colombia. \\ § Pediatra-Hemato-Oncólogo. Universidad Nacional de Colombia, Docente de la Fundación Universitaria de Ciencias de la Salud. \\ " Médico General. Universidad del Rosario, Bogotá, Colombia. \\ Hospital Infantil Universitario de San José, Bogotá, Colombia.
}

\section{RESUMEN}

La infección grave por Listeria monocytogenes en pacientes inmunocompetentes es infrecuente; en pediatría cobra relevancia durante la etapa neonatal y en una persona inmunocomprometida. Las manifestaciones en el sistema nervioso central (SNC) son encefalopatía, cuadro meníngeo, abscesos cerebrales o romboencefalitis. En la mayoría de las ocasiones el desenlace conlleva una alta morbimortalidad cuando no son diagnosticados a tiempo. Se presenta el caso de una paciente escolar con leucemia aguda linfoblástica de precursores $B$ de riesgo alto de reciente diagnóstico, quien presentó cuadro de neutropenia febril en el contexto de quimioterapia de inducción; posteriormente desarrolló crisis convulsivas de características focales que rápidamente evolucionaron a estado epiléptico, ameritando avanzar al manejo de tercera línea (coma inducido). La imagen por resonancia magnética (IRM) cerebral demostró áreas de realce parenquimatoso frontal superior izquierdo, compromiso de brazo posterior de cápsula interna derecha y lesión talámica ipsilateral, con sospecha de absceso. En la institución se contaba con prueba molecular de múltiples patógenos meníngeos (FilmArray ${ }^{\circledR}$ Biomerieux) siendo ésta positiva para $L$. monocytogenes, lo que determinó el tratamiento temprano dirigido con ampicilina a dosis altas, con evolución clínica favorable. Se discuten los aspectos más importantes de la infección de SNC por este patógeno, dígase epidemiológicos,
ABSTRACT

Severe infection by Listeria monocytogenes in immunocompetent patients is uncommon; in pediatrics it becomes relevant during the neonatal stage and in immunocompromised patients. Its manifestations in the central nervous system (CNS) are encephalopathy, meningeal symptoms, abscesses or rhombencephalitis. In the majority of cases, the outcome entails a high morbidity and mortality if a timely diagnosis is not made. The case of a school-aged female patient is presented. She had been newly diagnosed with high-risk $B$ precursor acute lymphoblastic leukemia, and presented with febrile neutropenia in the context of induction chemotherapy, she subsequently developed focal seizures that rapidly progressed to status epilepticus requiring thirdline management (induced coma). Brain magnetic resonance imaging (MRI) demonstrated areas of left upper frontal parenchymal enhancement, involvement of the posterior arm of the right internal capsule and ipsilateral thalamic lesion, suspicious of abscess. The institution had molecular tests for multiple meningeal pathogens (FilmArray ${ }^{\circledR}$ Biomerieux), one of which was positive for $\mathrm{L}$. monocytogenes, this determined early targeted treatment with high-dose ampicillin, with favorable clinical evolution. The most important aspects of CNS infection by this pathogen are discussed: epidemiological,

Citar como: Valderrama CA, Navarro PN, Lopera MJ, Castañeda CL. Neuroinfección por Listeria monocytogenes en una paciente pediátrica con leucemia aguda linfoblástica de reciente diagnóstico. La relevancia del diagnóstico precoz. Rev Latin Infect Pediatr. 2021; 34 (3): 142-147. https://dx.doi.org/10.35366/102237

Recibido: 22-10-2020. Aceptado: 03-12-2020.

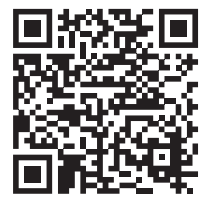


Rev Latin Infect Pediatr. 2021; 34 (3): 142-147

fisiopatológicos, imagenológicos y terapéuticos, con el fin de servir como guía para casos con comportamientos similares.

Palabras clave: Leucemia, neuroinfección, L. monocytogenes, ampicilina.

\section{INTRODUCCIÓN}

En pacientes oncológicos, la neutropenia es definida como un conteo de neutrófilos absolutos menor a $500 \mathrm{cel} / \mathrm{mm}^{3}$, es una complicación frecuente en los niños con cáncer y más aún en aquéllos que se encuentran en manejo quimioterapéutico. Cuando la neutropenia se asocia a fiebre, el paciente se encuentra en el contexto de una neutropenia febril, entidad relacionada con tasas de mortalidad del 30 al $50 \%$ en aquéllos que no son tratados con el antimicrobiano adecuado en las primeras 48 horas, ${ }^{1}$ mientras que en los que reciben atención temprana y dirigida, la tasa de mortalidad es tan baja como del 2-3\%., ${ }^{2,3}$ Aunque los agentes etiológicos en esta entidad son diversos, se reconoce que la etiología bacteriana es la más frecuente, lográndose identificar el germen en apenas el 20\% de los casos. ${ }^{4}$ Los microorganismos involucrados son dependientes de factores relacionados con la epidemiología local, el grado de inmunocompetencia del individuo, la presencia de dispositivos invasivos, entre otros. Históricamente, en los años ochenta del siglo pasado, los organismos aislados con mayor frecuencia en pacientes con neutropenia febril eran los gram negativos $y$, desde hace dos décadas, los más frecuentemente reportados son gram positivos, alcanzando cifras de hasta $16-70 \%$ en las diferentes series. ${ }^{1}$

Listeria monocytogenes es un bacilo grampositivo intracelular facultativo, catalasa positiva, con actividad beta hemolítica en el medio de cultivo agar sangre. En los seres humanos los serotipos asociados a infección son los 1/2a, 1/2b y $4 a$. Su presentación en brotes se ha asociado al consumo de alimentos contaminados. ${ }^{4}$ En 1985 , se documentó un brote masivo por esta bacteria en Los Ángeles, California, Estados Unidos de América, con 142 incidencias y 28 defunciones, el cual se atribuyó a una marca de quesos blandos contaminados con la bacteria. ${ }^{5}$ La enfermedad por $L$. monocytogenes no es la más común de las enfermedades transmitidas por alimentos, pero sí la que tiene tasas más altas de mortalidad, lo cual es secundario a sus factores de virulencia únicos. ${ }^{6}$ pathophysiological, imaging and therapeutic, in order to serve as a guide for cases with similar characteristics.

Keywords: Leukemia, neuroinfection, L. monocytogenes, ampicillin.

En un individuo inmunocompetente, la acción de dicho patógeno se presenta en la forma de una infección gastrointestinal autolimitada; ${ }^{2} \sin$ embargo, es un patógeno causante de meningitis en los grupos etarios ubicados en los «extremos de la vida" y en aquellos con compromiso de su sistema inmunológico. Es así que en pediatría este agente cobra relevancia durante la etapa neonatal, y, por otra parte, es responsable de cuadros catastróficos en el paciente inmunocomprometido.

La listeriosis es una infección oportunista del sistema nervioso central (SNC), con pocos reportes de eventos descritos en personas con cáncer. En un estudio publicado en 2003 por The University of Texas M.D. Anderson Cancer Center en Estados Unidos de América, se identificó una tasa de incidencia de L. monocytogenes en pacientes oncológicos de 42 por cada 100,000, con mayor presentación en aquellos del sexo masculino (59\%), con una media de edad de 55 años y se relacionó con neoplasia hematológica en el 59\% de los casos. ${ }^{7}$

\section{PRESENTACIÓN DEL CASO}

Paciente femenino de nueve años de edad, con diagnóstico reciente de leucemia linfoblástica aguda de precursores de células $B$ de alto riesgo, que se encontraba en poliquimioterapia en fase de inducción con prednisolona, vincristina, daunorrubicina y L-asparginasa. Después de su primer ciclo de tratamiento, presentó como complicación asociada un cuadro de neutropenia febril, con recuento absoluto de neutrófilos de 100 $\mathrm{cel} / \mathrm{mm}^{3}$ (Tabla 1), clínicamente debutó con dolor abdominal y diarrea. Se inició esquema antibiótico empírico con cefepima, con hemocultivos y coprocultivo sin aislamiento microbiológico; la ecografía abdominal no evidenció hallazgos sugestivos de colitis neutropénica. A partir del segundo día de administración de antibióticos se estabilizó clínicamente; sin embargo, al quinto día de antibioticoterapia presentó nuevo pico febril de 38.5 ${ }^{\circ} \mathrm{C}$ con persistencia de neutropenia, se decidió 
Rev Latin Infect Pediatr. 2021; 34 (3): 142-147

escalonar el tratamiento antibiótico a meropenem y vancomicina. Veinticuatro horas después, la menor presentó un episodio convulsivo de características focales, con alzas térmicas sostenidas; se ordenó tomografía axial computarizada (TAC) simple de cráneo (Figura 1A), que evidenció una hipodensidad cercana al área talámica derecha y brazo posterior de la cápsula interna. Se inició manejo de primera línea para estatus convulsivo, sin mejoría, por lo que se procedió con impregnación con fenitoína (PHT); considerando la quimioterapia recibida, se sospechó inicialmente trombosis cerebral relacionada con el uso de L-asparginasa, fue trasladada a la unidad de cuidados intensivos pediátricos (UCIP).

La niña tuvo deterioro neurológico y pocas horas después presentó recurrencia de crisis, instaurándose un estatus convulsivo focal refractario, ameritando manejo de tercera línea para éste (coma inducido). La paciente tenía un análisis citológico de líquido cefalorraquídeo (LCR) de una semana previa, que descartaba compromiso tumoral en el sistema nervioso central (Tabla 1). Se realizó una punción lumbar durante la primera hora de atención en la UCIP, solicitando un estudio citoquímico y microbiológico de LCR, con hallazgo de pleocitosis linfocítica con hipoglucorraquia e hiperproteinorraquia y detección de Listeria monocytogenes en Film-Array panel meníngeo. Se dirigió el tratamiento antibiótico a ampicilina con una duración total de 28 días. La imagen cerebral por resonancia magnética (IRM) (Figura 1B-D) mostró áreas de realce parenquimatoso frontal superior izquierda, en la cápsula interna derecha y en el tálamo ipsilateral, en esta última localización con evidencia de imagen ovalada de $24 \mathrm{~mm}$, con restricción al centro en la difusión y realce anular, asociada a restos hemorrágicos, con sospecha de absceso, y realce leptomeníngeo difuso supra- e infratentorial.

Tabla 1: Principales estudios realizados en el paciente.

Estudio

Hemograma inicial

Hemograma a las 24 horas

Proteína $\mathrm{C}$ reactiva

Ecocardiograma

Estudio citológico de LCR por punción lumbar inicial una semana antes

Estudio citológico de LCR por punción lumbar durante deterioro neurológico

Detección de múltiples patógenos por reacción en cadena de polimerasa meníngeo FilmArray ${ }^{\circledR}$ (Biomerieux)

Tomografía axial computada de cráneo

IRM cerebral

Electroencefalograma

\section{Resultados}

Leucocitos: $230 \mathrm{cel} / \mathrm{mm}^{3}$, neutrófilos absolutos: $0 \mathrm{cel} / \mathrm{mm}^{3}$, linfocitos absolutos: $170 \mathrm{cel} /$ $\mathrm{mm}^{3}$, hemoglobina: $7.1 \mathrm{~g} / \mathrm{dL}$, hematocrito: $20.4 \%$, plaquetas: $55.100 \mathrm{~mm}^{3}$

Leucocitos: $270 \mathrm{cel} / \mathrm{mm}^{3}$, neutrófilos absolutos: $0 \mathrm{cel} / \mathrm{mm}^{3}$, linfocitos absolutos: $200 \mathrm{cel} /$ $\mathrm{mm}^{3}$, monocitos absolutos: $30 \mathrm{cel} / \mathrm{mm}^{3}$, hemoglobina: $7.4 \mathrm{~g} / \mathrm{dL}$, hematocrito: $22.5 \%$, plaquetas: $32.700 \mathrm{~mm}^{3}$

$167.9 \mathrm{mg} / \mathrm{L}$

1. Corazón de estructura y función normal

2. No hay signos de cardiotoxicidad

3. No derrames, no masas

4. Sin signos de hipertensión pulmonar

5. Buena función sistólica biventricular

Glucosa $60 \mathrm{mg} / \mathrm{dL}$, proteínas $43 \mathrm{mg} / \mathrm{dL}$, 0 leucocitos $/ \mathrm{mm}^{3}$

Presión de apertura $12 \mathrm{~mm} \mathrm{H}_{2} \mathrm{O}$

Glucosa $28 \mathrm{mg} / \mathrm{dL}$, proteínas $307 \mathrm{mg} / \mathrm{dL}, 50$ eritrocitos $/ \mathrm{mm}^{3}, 65 \%$ crenados, 65 leucocitos/ $\mathrm{mm}^{3}$, PMN 8\%, linfocitos $77 \%$

Presión de apertura $26 \mathrm{~mm} \mathrm{H}_{2} \mathrm{O}$

Positivo para Listeria monocytogenes

Hipodensidad cercana al área talámica derecha y brazo posterior de la cápsula interna Áreas de realce parenquimatoso frontal superior izquierda, en la cápsula interna derecha y en el tálamo ipsilateral, en esta última localización con evidencia de imagen ovalada de $24 \mathrm{~mm}$, con restricción central en la difusión y realce anular Anormal, trazado encefalopático + epileptogénico temporal izquierdo de alta frecuencia 
Rev Latin Infect Pediatr. 2021; 34 (3): 142-147

Figura 1:

A) Tomografía axial computarizada simple de cráneo, donde se observa hipodensidad cercana al área talámica derecha y brazo posterior de la cápsula interna (flecha). B) Imagen cerebral por resonancia magnética, en corte axial secuencia DWI (Diffusion-weighted Magnetic Resonance Imaging) con restricción en la misma zona. C y D) Imagen cerebral por resonancia magnética, corte axial, secuencia T1 con contraste, con evidencia de imagen ovalada de $24 \mathrm{~mm}$ (flecha) y realce anular, así como de forma difusa, a nivel leptomeníngeo supra e infratentorial comprometiendo cisternas de la base. Cortesía: Servicio de Radiología, Hospital Infantil Universitario de San José. Bogotá, Colombia.
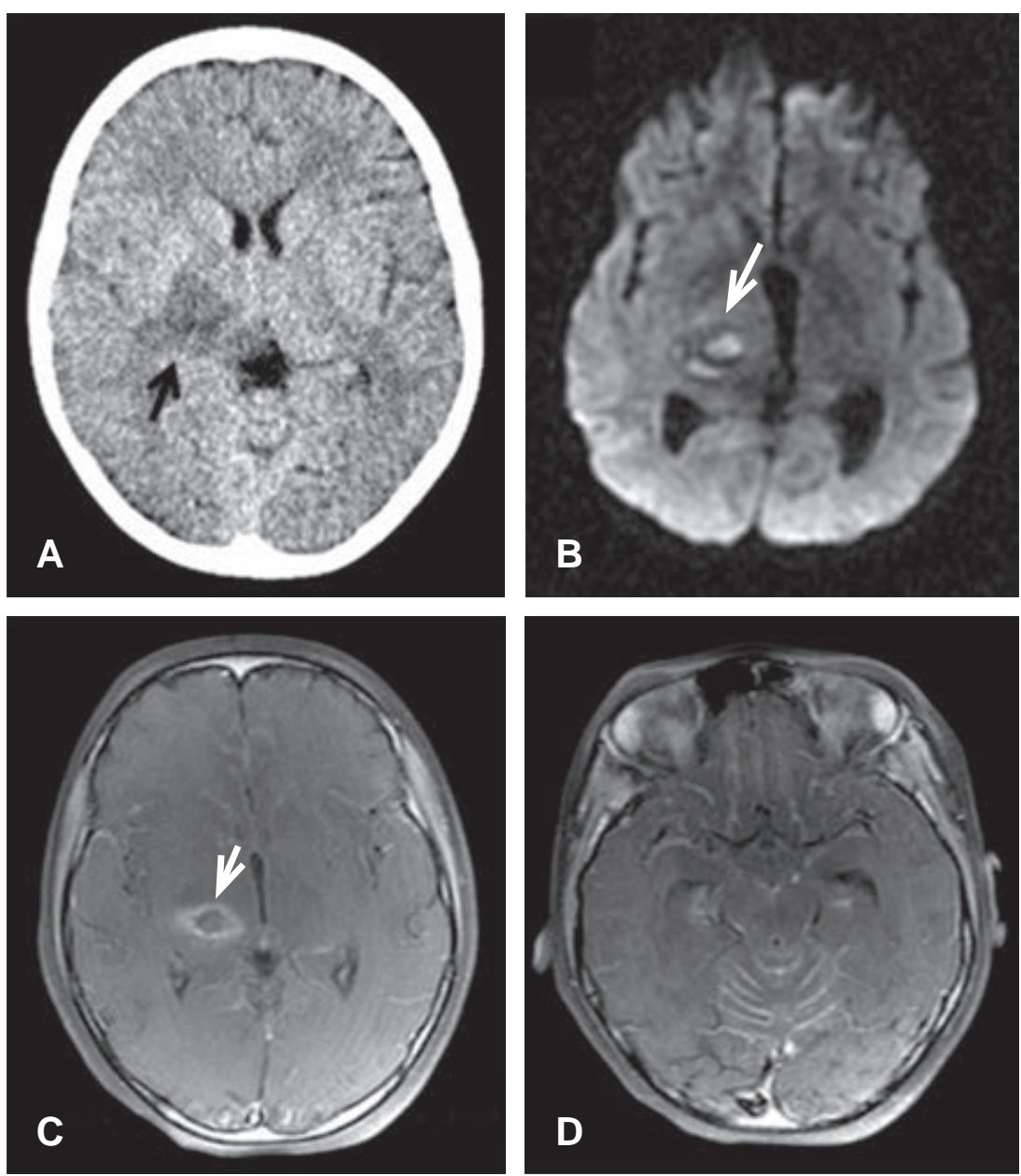

\section{DISCUSIÓN}

La evolución de la pequeña fue fluctuante, su electroencefalograma (EEG) reportó un trazado encefalopático con foco epileptogénico temporal izquierdo de alta frecuencia, a lo largo de su convalecencia se controló el estatus convulsivo y se cambió la medicación antiepiléptica a levetiracetam (LEV). Durante el tiempo de tratamiento mostró mejoría clínica paulatina, logrando retirarse la ventilación mecánica. Entre las secuelas de la neuroinfección por L. monocytogenes se documentó hemiparesia faciocorporal derecha de predominio crural, con alteración en los movimientos oculares por limitación en los movimientos sacádicos y a comando, asociada a una oftalmoparesia incompleta de pares craneales III, IV y VI que mejoró sustancialmente con el tiempo, así como un trastorno expresivo del lenguaje. Una vez resuelta la neuroinfección se continuó su tratamiento oncológico (quimioterapia) y se instauró su rehabilitación integral.
El caso presentado debutó con un cuadro de neutropenia febril y síntomas digestivos, con una posible asociación a colitis neutropénica que fue descartada; durante su evolución presentó detrimento en el sistema nervioso central, manifestado por crisis convulsivas sintomáticas con posterior estatus convulsivo focal refractario. El compromiso cerebral fue global, afectando la vía piramidal, pares craneales, meninges y tálamo, como se observó radiológicamente; a pesar del gran deterioro leptomeníngeo, no causó la romboencefalitis clásica ni los signos de disfunción a nivel de tallo cerebral, que suele ser un escenario reportado en la literatura ${ }^{8}$ y que está asociado a una alta morbimortalidad.

El cuadro de neuroinfección por L. monocytogenes puede ser insidioso e inespecífico, especialmente en pacientes inmunosuprimidos, por esta razón debería 
ser una consideración diagnóstica en los centros oncológicos, pues la falta de reconocimiento del conjunto de signos y síntomas y su progresión sin tratamiento oportuno puede ser letal. La listeriosis tiene una importante mortalidad en grupos de riesgo, dígase embarazadas, ancianos, neonatos o población inmunosuprimida. Aproximadamente un $30 \%$ de los casos de listeriosis no perinatal resulta en infección del SNC, y de éstos, el $13.7 \%$ desarrolla secuelas neurológicas; 9 con una mortalidad reportada de hasta $20 \%$ si no se inicia terapia antibiótica de forma oportuna. ${ }^{10}$ Entre los factores de riesgo identificados en este caso, además del inmunocompromiso, está que la niña y su familia solían consumir alimentos en locales callejeros de su entorno, lo que pudo estar estrechamente relacionado con la transmisión alimentaria de L. monocytogenes.

El estudio molecular para detección de múltiples patógenos por reacción de cadena de polimerasa (PCR) (FilmArray ${ }^{\circledR}$ Biomerieux) en líquido cefalorraquídeo (LCR), que se realizó en esta institución, fue pieza fundamental en el direccionamiento del tratamiento antibiótico. Esta prueba permite la identificación en una hora de catorce patógenos causantes de meningitis, los cuales se resumen en la Tabla 2.

Sin embargo, no en todas las instituciones se cuenta con pruebas de biología molecular que logren el dictamen etiológico de neuroinfección; razón por la cual, en las situaciones en que se sospeche etiología bacteriana, y principalmente en aquellos pacientes oncológicos con neutropenia febril que cursan con cuadros de romboencefalitis, el tratamiento antibiótico empírico debe incluir un agente antipseudomónico como la cefepima, sumado a ampicilina, ya que L. monocytogenes tiene una resistencia natural a las cefalosporinas; y una vez que se cuente con el resultado del cultivo de fluido cefalorraquídeo y un antibiograma se podrá dirigir la antibioticoterapia.
Respecto al análisis de las neuroimágenes, la neurolisteriosis cursa con una presentación neurorradiológica poco específica, pudiéndose asociar con romboencefalitis y abscesos cerebrales; sin embargo, la ausencia de estos hallazgos no descarta la infección por este microorganismo.

La imagen inicial (TAC de cráneo) del caso clínico planteó distintos diagnósticos diferenciales, complementariamente, en la IRM cerebral se encontraron hallazgos compatibles con un proceso inflamatorio leptomeníngeo y parenquimatoso, asociado a un realce perilesional con medio de contraste y probables áreas de transformación hemorrágica, por lo que se pensó en un efecto adverso de la L-asparaginasa, ya que este antineoplásico puede ocasionar eventos trombóticos que son en su mayoría venosos con o sin embolismo en el sistema nervioso central. La depleción de asparagina causada por la terapia con L-asparaginasa se asocia a una reducción en la síntesis de proteínas involucradas en la hemostasis, aumentando el riesgo de eventos trombóticos, los cuales tienen una incidencia de entre el 1-36\% dependiendo del grupo etario; éstos predominan en extremidades superiores y tienen asociación específica con trombosis de senos venosos cerebrales en niños. ${ }^{11}$ En una serie de casos basados en el estudio Multicentric Observational NAtional Study on LISteriosis and ListeriA (MONALISA) (Francia), en donde se evaluaron eventos de listeriosis (con cultivo positivo), se encontró que además de los hallazgos mencionados, es común encontrar imágenes hemorrágicas. ${ }^{10}$

Otro diagnóstico diferencial es la infiltración tumoral del SNC, sin embargo, la menor contaba con un examen citológico de líquido cefalorraquídeo de dos semanas previas que excluía compromiso a este nivel. La resolución favorable de las lesio-

Tabla 2: Detección de múltiples patógenos en líquido cefalorraquídeo por reacción en cadena de polimerasa.

Bacterias

Escherichia coli K1 Haemophilus influenzae Listeria monocytogenes Neisseria meningitidis Streptococcus agalactiae Streptococcus pneumoniae
Virus

Citomegalovirus

Enterovirus

Virus herpes simple 1

Virus herpes simple 2

Herpesvirus humano 6

Parechovirus humano

Virus varicela-zóster
Levaduras

Cryptococcus neoformans/gattii 
Rev Latin Infect Pediatr. 2021; 34 (3): 142-147

nes con antibioticoterapia contribuyó a aclarar el dictamen. Aunque la tuberculosis meníngea es un diagnóstico diferencial en el contexto de una paciente inmunocomprometida con meningitis, con hallazgo de pleocitosis linfocítica con hipoglucorraquia e hiperproteinorraquia en LCR y neuroimagen con realce leptomeníngeo y compromiso gangliobasal, la presentación aguda del cuadro y la detección de $L$. monocytogenes mediante prueba de biología molecular permitieron excluir Mycobacterium tuberculosis como agente etiológico.

En una serie de 71 casos de neurolisteriosis se reportaron imágenes nodulares en $14 \%$, disminución de la sustancia blanca en 59\%, y no se encontró relación entre la presentación radiológica y el número de comorbilidades relacionadas con la terapia de inmunosupresión. En pacientes con imágenes de nódulos, abscesos intraparenquimatosos encefálicos o imágenes no específicas en sustancia blanca se reportó menor supervivencia a los tres meses. ${ }^{10}$

\section{CONCLUSIONES}

La sospecha diagnóstica de neuroinfección por $L$. monocytogenes en pacientes inmunosuprimidos permite iniciar un tratamiento de forma precoz, con el propósito de mitigar las consecuencias y secuelas en el neurodesarrollo. Es importante tener en mente a los grupos en riesgo de contraer esta infección, como son los neonatos y los inmunocomprometidos. Si bien es cierto que la neurolisteriosis es poco frecuente en la actualidad, las tasas de mortalidad son altas si no se instaura el manejo de elección $(20 \%) .{ }^{10}$ En el caso presentado, se brindó inicialmente un esquema antibiótico de amplio espectro, que fue dirigido a monoterapia con ampicilina una vez que se contó con el resultado de la prueba molecular donde se identificó ese microorganismo en el LCR. Fue de esta manera que se realizó el control de la infección y se logró un resultado favorable en la paciente, quien ahora se encuentra en remisión de la leucemia y en terapia de rehabilitación integral.

\section{REFERENCIAS}

1. Paganini $H$, Santolaya PME, Álvarez M, Araña RM, Arteaga BR, Bonilla A et al. Diagnóstico y tratamiento de la neutropenia febril en niños con cáncer: Consenso de la Sociedad Latinoamericana de Infectología Pediátrica. Rev Chil Infect. 2011; 28: 10-38.

2. White L, Ybarra M. Neutropenic fever. Emerg Med Clin North Am. 2014; 32 (3): 549-561.

3. Rivera-Salgado D, Valverde-Muñoz K, Ávila-Agüero M. Neutropenia febril en niños con cáncer: manejo en el servicio de emergencias. Rev Chil Infect. 2018; 35 (1): 62-71.

4. Rogalla D, Bomar PA. Listeria Monocytogenes. $2020 \mathrm{Jul} 10$. In: StatPearls [Internet]. Treasure Island (FL): StatPearls Publishing; 2021.

5. Choi MH, Park YJ, Kim M, Seo YH, Kim YA, Choi JY et al Increasing incidence of listeriosis and infection-associated clinical outcomes. Ann Lab Med. 2018; 38 (2): 102-109.

6. Jordan K, McAuliffe O. Listeria monocytogenes in foods. Adv Food Nutr Res. 2018; 86: 181-213.

7. Rivero GA, Torres HA, Rolston KVI, Kontoyiannis DP. Listeria monocytogenes infection in patients with cancer. Diagnostic Microbiol Infect Dis. 2003; 47 (2): 393-398.

8. Illanes DS, Araya CP, Ferrer DS, Cartier RL, Bravo MM, Castillo CS. Romboencefalitis: una forma de infección por Listeria monocytogenes en el sistema nervioso central. Rev Méd Chile 2003; 131: 921-928.

9. Maertens NCM, Devleesschauwer B, Angulo FJ, Verbeke G, Haagsma J, Kirk M et al. The global burden of listeriosis: a systematic review and meta-analysis. Lancet Infect Dis. 2014; 14 (11): 1073-1082.

10. Charlier C, Poirée S, Delavaud C, Khoury G, Richaud $\mathrm{C}$, Leclercq A et al. Imaging of human neurolisteriosis: a prospective study of 71 cases. Clin Infect Dis. 2018; 67 (9): 1419-1426.

11. Goyal G, Bhatt VR. L-asparaginase and venous thromboembolism in acute lymphocytic leukemia. Future Oncol. 2015; 11 (17): 2459-2470.

Financiamiento: Por los autores.

Conflicto de intereses: Ninguno.

Correspondencia:

Abel Valderrama Cisneros

E-mail: neuropediatria@hospitalinfantildesanjose.org.co 